\title{
Convenios Marco y su Aplicación en las Universidades Públicas Chilenas: Periodo 2015-2016
}

\author{
Patricia C. Huerta* y Héctor G. Gaete \\ Universidad del Bío-Bío, Avenida Collao 1202, Concepción-Chile. (e-mail: phuerta@ubiobio.cl; \\ hgaete@ubiobio.cl) \\ * Autor a quien debe ser dirigida la correspondencia
}

Recibido Jul. 4, 2018; Aceptado Sep. 25, 2018; Versión final Nov. 19, 2018, Publicado Abr. 2019

\begin{abstract}
Resumen
Este artículo analiza los proyectos estratégicos institucionales, denominados Convenios Marco, los cuales a partir del año 2015 representan una nueva modalidad de financiamiento para las universidades públicas chilenas. Por ello el objetivo del artículo consiste en analizar la instalación del convenio marco sobre la acreditación institucional, planificación estratégica y el aseguramiento de la calidad, de las universidades públicas. La metodología utilizada es análisis descriptivo y análisis de correspondencias múltiples, para el periodo 2015-2016. Los resultados del estudio permiten apreciar que existe una asociación entre las áreas de acreditación institucional, la planificación estratégica y en menor medida el aseguramiento de la calidad, con los proyectos convenio marco. Se concluye que los impactos del convenio marco deben ser estudiados a largo plazo, para verificar la permanencia en el tiempo del mejoramiento de la calidad en las instituciones de educación superior.
\end{abstract}

Palabras clave: universidad; planificación; acreditación; educación; calidad

\section{Framework Conventions and their Application in Chilean Public Universities: Period 2015-2016}

\begin{abstract}
This article analyzes the strategic institutional projects called Framework Conventions, which since 2015 represent a new form of financing for Chilean public universities. Therefore, the objective of the article consists of analyzing the installation of the framework conventions on institutional accreditation, strategic planning and quality assurance of public universities. The methodology used includes descriptive analysis and analysis of multiple correspondences, for the period 2015-2016. The results of the study allow us to appreciate that there is an association between the areas of institutional accreditation and strategic planning, and in lesser extent quality assurance, with the framework convention projects. It is concluded that, the impacts of the framework conventions should be studied in the long term, to verify the permanence over time of the improvement of quality in higher education institutions.
\end{abstract}

Keywords: university; planning; accreditation; education; quality 


\section{INTRODUCCIÓN}

La educación superior ha sido considerada tradicionalmente como un bien público que debería ser provisto y financiado esencialmente por el Estado (Casani et al., 2014: 48), sin embargo, ese financiamiento no es suficiente, debido a las diversas necesidades que existen en el ámbito de la educación superior, tales como el sistema de ayudas, compuesto por becas y préstamos, y el programa de gratuidad que involucra una gran cantidad de recursos públicos y que pretenden incrementarse en los próximos años, entre otras necesidades del sistema de educación superior. Por esta razón, y con la finalidad de mejorar la calidad y el acceso a la educación superior, el aporte en educación en Chile ha aumentado en los últimos años, específicamente, según la Contraloría General de la República, el gasto en educación el año 2017 fue de MMUS\$ 3.076, un 7,8\% superior al año anterior, y de acuerdo al informe 2017 de la Organización para la Cooperación y el Desarrollo Económico (OCDE), el gasto en educación terciaria como porcentaje del PIB en Chile fue del 2,3\% el año 2014, siendo del 1,6\% en la OCDE, sin embargo cuando se considera el gasto en educación terciaria pública éste representa el 1,2\% del PIB en Chile y el 1,3\% en la OCDE. Por lo anterior, se requiere de la colaboración entre las universidades (Spodarczyk y Szelagowska, 2015) para poder responder a las necesidades de educación, tal como la cooperación que existe entre universidades de Europa y Latinoamérica (García y Henry 1993), o los ejemplos de cooperación entre las universidades y la industria (Lukac y Chatzimichailidou, 2017), siendo varios los beneficios de la cooperación (Keller, 2012). En este sentido es importante reconocer que detrás de estas relaciones existen organismos que impulsan esta cooperación, tales como los Programas Marco de la Unión Europea, el Programa Iberoamericano de Ciencia y Tecnología para el Desarrollo (CITED), el Mercosur Educativo, las políticas de internacionalización emitidas por la Alianza del Pacífico, el Estado de Chile, al igual que el Banco Mundial que es el organismo internacional que promueve los Convenios Marco.

Es por ello que los proyectos de reforma educacional en el mundo nos obligan a reflexionar sobre la relevancia de incorporar nuevos mecanismos que permitan fortalecer las instituciones de educación superior y que fomenten la cooperación entre ellas, con la finalidad de propiciar la formación de capital humano avanzado, y de esta forma, poder enfrentar los desafíos que posee la sociedad. Siendo un desafío la cooperación internacional (Kapliienko, 2016; Leal, 2017) debido a las ventajas de utilizar a las universidades como socio de cooperación (Rõigas et al., 2018). Bajo este contexto el sistema de educación superior en Chile se encuentra vinculado a diversos mecanismos de financiamiento y desarrollo institucional (Araneda-Guirriman y Pedraja-Rejas, 2016; Araneda-Guirriman et al., 2018), que buscan aportar al crecimiento de las regiones y del país, al igual que a las instituciones de educación. Estos mecanismos tienen por finalidad propiciar una mayor y mejor calidad, cuyos resultados se observan, a través de los estándares de acreditación institucional. Por esta razón, y con la finalidad de impulsar un sistema de colaboración entre las universidades públicas, surge en Chile un mecanismo de financiamiento, único en el mundo, denominado convenio marco (CM), el cual se manifiesta a través de un proyecto estratégico que formula una universidad, de acuerdo a algunos lineamientos. Específicamente, el convenio marco es un instrumento del Ministerio de Educación (Mineduc), que coloca a disposición de las universidades públicas, para permitir el fortalecimiento institucional, el cual debe estar alineado a la planificación estratégica de la universidad.

De esta forma, el Ministerio realiza un acuerdo con las universidades para definir, un plan de fortalecimiento a cinco años, representado a través del plan plurianual 2016-2020. En particular, a nivel internacional no existe una forma de colaboración similar. Siendo lo más cercano los proyectos del ámbito de educación que el Banco Mundial lleva adelante en diferentes países (Post et al., 2004: 213), al igual que los estudios de cooperación que vinculan a las universidades con la industria (Keller, 2012: 58; Thamae et al., 2016; Yang, 2016) o los que vinculan a las empresas con la universidad (Pavlin, 2016; Pavlin et al., 2016; Ranga et al., 2016) a través de diferentes metodologías como el aprendizaje basado en problemas (Rossano et al., 2016) o el fomento de la cooperación para el desarrollo (De Francisco et al., 2017; Marques et al., 2016). En concreto, este mecanismo busca consolidar un estándar de calidad común para todas las universidades estatales, propiciando el trabajo cooperativo y la trasmisión de conocimiento, a través del trabajo en red que se establece, fortaleciendo de esta forma, la identidad pública que poseen por esencia las Universidades del Estado. A su vez, las instituciones pueden dirigir su quehacer y conocimiento como un aporte hacia el desarrollo de la comunidad y la unión de las universidades con sus regiones, con la finalidad de asegurar el crecimiento y desarrollo social y la oportunidad de fortalecer el trabajo entre las universidades públicas, lo que se traduce en mayor desarrollo de políticas de calidad, investigación y vinculación con el medio. Siendo un factor clave el rol que ejerce el gobierno para el éxito de la cooperación entre universidades (Zhimin et al., 2016).

Con el objetivo de analizar los proyectos estratégicos institucionales, a través de un nuevo mecanismo de financiamiento y desarrollo institucional, el presente estudio se estructura en los siguientes apartados: En primer lugar, se presentan las bases conceptuales de convenio marco, planificación estratégica y 
acreditación institucional y, en segundo lugar, se contrastan los planteamientos anteriores para proporcionar evidencia al respecto, a través del análisis de la instalación de los convenios marco sobre la acreditación institucional, planificación estratégica y el aseguramiento de la calidad, de las universidades públicas chilenas para el periodo 2015-2016. Por último, se presentan las conclusiones del estudio y las referencias utilizadas.

\section{CONVENIO MARCO PARA LAS UNIVERSIDADES PÚBLICAS}

El convenio marco comienza en Chile el año 2015, con el objetivo de elaborar un plan de fortalecimiento a cinco años, que se implementa anualmente en base a un proyecto estratégico institucional que presenta cada universidad pública. Los proyectos se elaboran alineados al plan estratégico institucional, considerando las políticas públicas en educación y, buscando fortalecer las capacidades institucionales. Sin embargo, Palma y Rodríguez (2017: 194-195), plantean que el convenio marco no proporciona recursos de libre disponibilidad, sino que las universidades del Estado se obligan a cumplir un plan plurianual, cuyos fines específicos son de interés del Ministerio de Educación.

Cada proyecto se estructura en base a una propuesta formulada inicialmente por el Mineduc, la cual es trabajada posteriormente por cada universidad, e incluye los lineamientos para el trabajo en red. El proyecto de convenio marco base se elabora a partir de objetivos institucionales, objetivos en red, hitos, actividades, indicadores y medios de verificación. Siendo uno de los objetivos la elaboración del plan plurianual, el cual se formula en torno a determinadas acciones dentro en los siguientes ejes: Fortalecimiento institucional; Fortalecimiento de la investigación, innovación y postgrado; Fortalecimiento en temas de Vinculación con el Medio, en forma articulada con las otras Universidades del Estado. Finalmente, también se incluye un eje de fortalecimiento de la formación inicial docente (FID).

En particular, en el primer convenio marco, el eje de fortalecimiento institucional se orientaba al mejoramiento de las capacidades institucionales de recolección y de análisis de datos, el fortalecimiento de las capacidades institucionales de planificación estratégica y aseguramiento de la calidad, al igual que el potenciar las estructuras de soporte de las diferentes funciones institucionales, y el análisis y reducción de brechas internas de la institución. Lo anterior, para lograr una gestión eficiente y homogénea en las diferentes unidades académicas y administrativas. En cambio, el eje de vinculación con el medio (VCM), consistía en ser parte activa de la red de universidades públicas, desarrollando acciones conjuntas, al igual que implementando un plan de movilidad académica y estudiantil entre las universidades del Estado, al igual que otras acciones definidas por cada institución. A su vez, el eje de investigación e innovación se orientaba a aumentar la productividad científica, innovación, transferencia tecnológica (TT), investigación, desarrollo e innovación $(\mathrm{I}+\mathrm{D}+\mathrm{i})$ y capacidades de investigación al interior de las instituciones.

Relevante en la formulación de los convenios marco es la definición del equipo directivo, el cual debe estar conformado por autoridades de alta jerarquía, con la finalidad de asegurar el liderazgo y compromiso institucional y así velar por el impacto de cada proyecto, al igual que su instalación y transversalidad. También se requiere de un comité ejecutivo, el cual debe estar constituido por autoridades y profesionales a cargo del desarrollo de la propuesta, quienes deben estar comprometidos en su ejecución, y son los responsables del buen funcionamiento de la iniciativa. Adicionalmente, forma parte de la ejecución de los proyectos, el responsable de la Unidad de Coordinación Institucional, quien es el encargado de apoyar para el desarrollo financiero de los CM adjudicados. Por último, todo proyecto estratégico, debía considerar un organigrama funcional que represente la forma cómo se inserta en la orgánica institucional, considerando sus relaciones de dependencia y articulación. En la figura 1 se muestra un modelo de gestión de los convenios marco de una universidad pública, es decir, una universidad estatal.

Es importante conocer la forma en la cual se componen los recursos de convenios marco, los cuales variaron en el periodo 2015-2016. En particular, el año 2015 la Ley №20.798 de presupuestos del sector público involucró un fondo a repartir para las universidades estatales por $\mathrm{M} \$ 34.080 .000$, que consideraba una base histórica y recursos adicionales para los convenios marco 2015 (CM 2015). El año 2016 la Ley № 20.882 de presupuestos del sector público involucró un monto total de $\mathrm{M} \$ 46.539 .593$ para convenio marco, 40\% distribuido en el primer semestre (CM 2016_1) y el 60\% restante en el segundo semestre (CM 2016_2). En cambio, el año 2017 la Ley 20.981 consigna recursos por $M \$ 57.826 .374$, distribuidos en igual porcentaje que el año anterior, sin embargo, al 11 de octubre 2017, fecha de cierre de ese estudio, los proyectos 2017 no se encuentran disponibles en la página web del Ministerio de Educación de Chile. Por otra parte, de acuerdo a las bases de los convenios marco, éstos deben estar alineados a la planificación estratégica de la universidad, $y$, por ende, al proceso de acreditación institucional, por ello es necesario revisar sus conceptos claves, los cuales se presentan a continuación. 


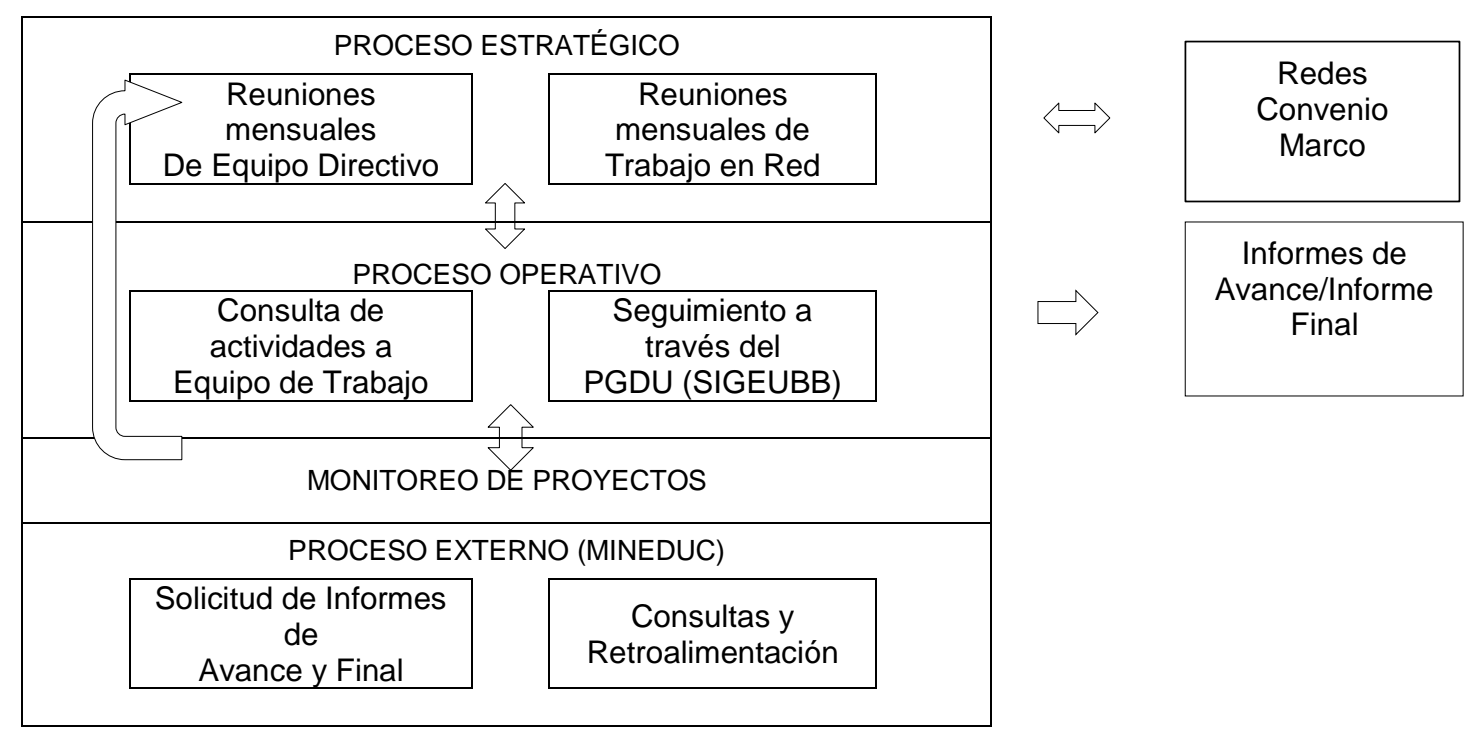

Fig. 1: Modelo de gestión de los convenios marco de la Universidad del Bío-Bío

\section{PLANIFICACIÓN ESTRATÉGICA Y ACREDITACIÓN INSTITUCIONAL}

La planificación estratégica es un instrumento que puede ser aplicado a todo tipo de organizaciones (Cuesta, 2012: 36) y por ello ha sido objeto de diversos estudios. Siendo el sector de educación superior, el que utiliza la planificación estratégica para guiar a la institución, de acuerdo a los objetivos que la comunidad desea alcanzar en el mediano y largo plazo. Lo anterior, se produce en las universidades públicas, porque en ellas el proceso de planificación se caracteriza por ser un proceso participativo y de búsqueda de consensos, en torno a los desafíos de futuro que se persigan. Vinculado al proceso de planificación institucional, se encuentra el proceso de acreditación, el cual ha cobrado gran relevancia a nivel internacional en diversos países, tal como lo demuestran los estudios de Acosta (2015) y Mollis (2014). Y en particular en Chile la acreditación ha sido objeto de diversas investigaciones, tal como los estudios de Cancino y Schmal (2014) y Venables y Van Gastel (2014), entre otros.

En efecto, la acreditación en Chile es un proceso relevante para la mayoría de las Universidades, porque evidencia la calidad de las instituciones, de las carreras, y de los programas que las instituciones dictan. Donde la Ley $\mathrm{N}^{\circ} 20.129$ del Sistema Nacional de Aseguramiento de la Calidad comprende el proceso de acreditación institucional, proceso que involucra el análisis de los mecanismos existentes en las instituciones autónomas de educación superior, para asegurar su calidad, considerando tanto la existencia de dichos mecanismos, como su aplicación y resultados. En concreto, las instituciones pueden acreditarse en dos áreas mínimas que tienen un carácter obligatorio, como son la docencia de pregrado y gestión institucional, pudiendo acceder a áreas opcionales, como son investigación, docencia de postgrado y vinculación con el medio, es decir, las instituciones de educación superior se pueden acreditar por un máximo de 5 áreas y hasta 7 años. Siendo la Comisión Nacional de Acreditación (CNA), el organismo autónomo que goza de personalidad jurídica y patrimonio propio, cuya función es verificar y promover la calidad de las Universidades, Institutos Profesionales y Centros de Formación Técnica autónomos, de las carreras y programas. En definitiva, las instituciones se someten a la acreditación para certificar el cumplimiento del proyecto que posee la institución y la existencia, aplicación y resultados de mecanismos eficaces de autorregulación y de aseguramiento de la calidad, al interior de las mismas.

Las instituciones de educación superior, y dentro de ellas las universidades, se someten a estos procesos impulsados con la finalidad de certificar su calidad, debido a que esta certificación les permite poder acceder a fondos estatales $\mathrm{y}$, al reconocimiento de la comunidad sobre su calidad y los beneficios que pueden ofrecer a sus estudiantes por lograrla. A la vez, en Chile la Ley sobre Educación Superior plantea que la acreditación tiene un carácter obligatorio, sin embargo, tal como señala Martínez et al. (2017: 73), antes el proceso era voluntario, para las instituciones preocupadas por la calidad. Siendo la calidad, la principal preocupación que deben tener las instituciones en la ejecución de sus actividades, de manera que la acreditación se vuelva el resultado de la calidad implementada por ella y no sólo la búsqueda de una validación en el medio. A su vez, la calidad y los mecanismos utilizados para su verificación deberían estar considerados en el proceso de planificación estratégica y ser utilizados de manera transversal por la institución. Por lo tanto, calidad, acreditación y planificación, no son neutrales, sino más bien, son conceptos relacionados pero diferentes y por esta razón orientan las políticas públicas y la planificación de las instituciones de educación superior. En 
esta línea, Gómez et al. (2017: 65) en su estudio sobre acreditación institucional, plantean que se deben "definir procesos de autoevaluación y evaluación con reglas claras y estables, equitativas, no discrecionales y transparentes, que consideren la realidad del entorno y, que la calidad de la educación no debe responder a cambios en la gobernabilidad, la institucionalidad, ni tener sesgo político o ideológico".

Dada la argumentación anterior se presentan las siguientes hipótesis de investigación: Hipótesis 1: Los convenios marco contribuyen a la implementación de la planificación estratégica de las Universidades Públicas en Chile, caeteris paribus; e Hipótesis 2: Los convenios marco se asocian al fortalecimiento de las áreas de acreditación institucional y el aseguramiento de calidad, en las Universidades Públicas en Chile, caeteris paribus.

\section{METODOLOGÍA}

El presente estudio es de tipo descriptivo y cuantitativo, cuya fuente de información utilizada son, los proyectos estratégicos convenio marco que formulan las universidades públicas chilenas, proyectos publicados al 11 de octubre del año 2017, por el Departamento de Financiamiento Institucional de la División de Educación Superior del Ministerio de Educación en Chile, en su página web, para el periodo 2015-2016. En concreto, se aplica una técnica estadística cuantitativa, como es el análisis de correspondencias múltiples, y posteriormente se construyen variables cualitativas o categóricas para el análisis posterior.

Con esta información, se realiza un análisis identificando las áreas de la acreditación institucional, que se evidencian explícitamente en los objetivos específicos institucionales y/o hitos y/o actividades de los proyectos convenios marco, presentados por las universidades públicas al Ministerio de Educación. De manera similar, se analiza la asociación hacia la planificación estratégica y al aseguramiento de la calidad. Conceptos fuertemente relacionados pero diferentes, razón por la cual se consideraron por separado entre sí y de las áreas de acreditación institucional. Para ello, se revisó el proyecto CM 2015, CM 2016_1 y CM 2016_2, considerando sólo el apartado de compromisos institucionales y no los objetivos en red de cada proyecto, ni los objetivos generales, ni los anexos. Lo anterior, debido a que la esencia del proyecto se refleja en los elementos mencionados. Por ende, en esta etapa, no se consideran las consecuencias que genera la ejecución del objetivo, hito o actividad, de los conceptos analizados.

Además, de manera complementaria se estudió la dependencia directa de cada proyecto, a través del director y el director alterno. Sin embargo, en algunas instituciones el director alterno o subdirector de CM no se encontraba explícito en los proyectos, y en esos casos, se consideró el cargo que se menciona después del director de proyecto. Además, el director ejecutivo no fue considerado como alterno. Por otra parte, un cargo puede involucrar dos áreas, tal como las dos áreas reflejadas en la vicerrectoría de investigación y postgrado, entre otros. En definitiva, se consideraron para el análisis los nombres de los cargos que presenta el convenio marco. Sin embargo, cuando un cargo jerárquico representa a un área estudiada, pero el cargo en $\mathrm{CM}$ representa a otra área de acreditación, el área jerárquica sirvió para definir la asociación, y así no generar duplicación. Con la finalidad de observar si las áreas de acreditación institucional, planificación estratégica y aseguramiento de la calidad, se encuentran presentes en quienes lideran estos proyectos estratégicos, se analizó también el cargo de quienes conforman el comité directivo y el comité ejecutivo. En particular, la Formación Inicial Docente se asoció al área de docencia y no se consideró si dentro de sus objetivos contemplaba los conceptos analizados, porque sólo se refiere a pedagogías.

En definitiva, se aplica análisis descriptivo y análisis de correspondencias múltiples, la cual es una técnica descriptiva que se utiliza para representar gráficamente la frecuencia de aparición de más de dos variables cualitativas o categóricas. El utilizar esta técnica estadística multivariada permite representar y observar la estructura de relaciones que se generan entre las variables del estudio, mediante un mapa de posicionamiento, en donde cada categoría es ubicada en un punto y la distancia entre las distintas categorías brinda la posibilidad de analizar la relación entre ellas, siendo una técnica requerida para poder estudiar la relación entre variables categóricas presentes en este estudio. Para el análisis de la información se utilizó el programa estadístico SPSS 21.

\section{Población. Objeto de estudio y variables}

La población objeto de estudio la componen las universidades chilenas acreditadas por la CNA, dentro del periodo 2015-2016, y la muestra está compuesta sólo por las universidades públicas chilenas acreditadas en el mismo periodo. En la tabla 1 se muestran las instituciones de educación superior en Chile vigentes del Mineduc, información extraída de: www.mifuturo.cl al 30.06.2017. Sin embargo, se debe considerar que existen dos universidades públicas de reciente creación, razón por la cual no se encuentran acreditadas y por ello no se consideran en este estudio. En la tabla 2 se muestran las variables del estudio. 
Tabla 1: Población y muestra 2015-2016

\begin{tabular}{|l|c|c|c|}
\hline \multicolumn{1}{|c|}{ Categoría universidades } & Universidades acreditadas & Universidades no acreditadas & Total universidades \\
\hline Privadas & 30 & 13 & 43 \\
\hline Públicas & 16 & 2 & 18 \\
\hline Total & 46 & 15 & 61 \\
\hline
\end{tabular}

Tabla 2: Variables del Estudio

\begin{tabular}{|c|c|c|}
\hline Variable & Definición & Descripción de la variable \\
\hline $\begin{array}{l}\text { Tipo de director } \\
\text { del proyecto }\end{array}$ & $\begin{array}{l}\text { Instancia universitaria de la } \\
\text { cual depende el proyecto } \\
\text { convenio marco }\end{array}$ & $\begin{array}{l}\text { Variable categórica que toma el valor "1" cuando la unidad } \\
\text { encargada del proyecto convenio marco depende directamente de } \\
\text { Rectoría, toma el valor "2" cuando depende del Prorrector(a), el } \\
\text { valor " } 3 \text { " cuando depende de la Vicerrectoría y toma el valor "4" } \\
\text { cuando depende de una dirección. }\end{array}$ \\
\hline $\begin{array}{l}\text { Tipo de Director } \\
\text { alterno }\end{array}$ & $\begin{array}{l}\text { Instancia universitaria que } \\
\text { subroga al director del } \\
\text { proyecto }\end{array}$ & $\begin{array}{l}\text { Variable categórica que toma el valor "1" cuando la unidad } \\
\text { encargada de del proyecto convenio marco depende directamente } \\
\text { de Rectoría, toma el valor "2" cuando depende del Prorrector(a), } \\
\text { el valor "3" cuando depende de la Vicerrectoría y toma el valor " } 4 \text { " } \\
\text { cuando depende de una dirección. }\end{array}$ \\
\hline $\begin{array}{l}\mathrm{N}^{\circ} \text { áreas del } \\
\text { Proyecto }\end{array}$ & $\begin{array}{l}\text { Número de áreas de } \\
\text { acreditación institucional, } \\
\text { reflejadas en el proyecto }\end{array}$ & $\begin{array}{l}\text { Variable categórica que toma el valor " } 1 \text { " cuando el proyecto } \\
\text { posee } 1 \text { área, toma el valor " } 2 \text { " cuando posee } 2 \text { áreas, toma el } \\
\text { valor "3" cuando posee } 3 \text { áreas, toma el valor " } 4 \text { " cuando posee } 4 \\
\text { áreas y toma el valor " } 5 \text { " cuando posee } 5 \text { áreas. }\end{array}$ \\
\hline $\begin{array}{l}N^{\circ} \text { áreas en } \\
\text { comité directivo }\end{array}$ & $\begin{array}{l}\text { Número de áreas de } \\
\text { acreditación institucional, } \\
\text { reflejadas en el comité } \\
\text { directivo }\end{array}$ & $\begin{array}{l}\text { Variable categórica que toma el valor "1" cuando el comité } \\
\text { directivo posee } 1 \text { área, toma el valor "2" cuando posee } 2 \text { áreas, } \\
\text { toma el valor " } 3 \text { " cuando posee } 3 \text { áreas, toma el valor " } 4 \text { " cuando } \\
\text { posee } 4 \text { áreas y toma el valor " } 5 \text { " cuando posee } 5 \text { áreas. }\end{array}$ \\
\hline $\begin{array}{l}\mathrm{N}^{\circ} \text { áreas en } \\
\text { comité ejecutivo }\end{array}$ & $\begin{array}{l}\text { Número de áreas de } \\
\text { acreditación institucional, } \\
\text { reflejadas en el comité } \\
\text { ejecutivo }\end{array}$ & $\begin{array}{l}\text { Variable categórica que toma el valor "1" cuando el comité } \\
\text { ejecutivo posee } 1 \text { área, toma el valor " } 2 \text { " cuando posee } 2 \text { áreas, } \\
\text { toma el valor " } 3 \text { " cuando posee } 3 \text { áreas, toma el valor " } 4 \text { " cuando } \\
\text { posee } 4 \text { áreas y toma el valor " } 5 \text { " cuando posee } 5 \text { áreas. }\end{array}$ \\
\hline $\begin{array}{l}\text { Áreas de } \\
\text { Acreditación } \\
\text { Institucional }\end{array}$ & $\begin{array}{l}\text { Número de áreas } \\
\text { otorgados por la CNA, para } \\
\text { la Acreditación Institucional }\end{array}$ & $\begin{array}{l}\text { Variable categórica que toma el valor "1" cuando la universidad } \\
\text { posee } 2 \text { áreas, toma el valor "2" cuando posee } 3 \text { áreas, toma el } \\
\text { valor "3" cuando posee } 4 \text { áreas y toma el valor "4" cuando posee } \\
5 \text { áreas. }\end{array}$ \\
\hline $\begin{array}{l}\text { Años de } \\
\text { Acreditación } \\
\text { Institucional }\end{array}$ & $\begin{array}{l}\text { Número de años otorgados } \\
\text { por la CNA, para la } \\
\text { Acreditación Institucional }\end{array}$ & $\begin{array}{l}\text { Variable categórica que toma el valor "1" cuando la universidad } \\
\text { posee } 3 \text { años, toma el valor "2" cuando posee } 4 \text { años, toma el } \\
\text { valor "3" cuando posee } 5 \text { años, toma el valor "4" cuando posee } 6 \\
\text { años y toma el valor " } 5 \text { " cuando posee } 7 \text { años. }\end{array}$ \\
\hline $\begin{array}{l}\text { Planificación } \\
\text { reflejado en el } \\
\text { Proyecto }\end{array}$ & $\begin{array}{l}\text { Existencia del ámbito de } \\
\text { planificación, explícito en el } \\
\text { proyecto }\end{array}$ & $\begin{array}{l}\text { Variable dicotómica que toma el valor "1" cuando el proyecto } \\
\text { evidencia explícitamente que posee el ámbito de planificación y } \\
\text { toma el valor "0" en caso contrario. }\end{array}$ \\
\hline $\begin{array}{l}\text { Planificación } \\
\text { reflejado en el } \\
\text { comité directivo }\end{array}$ & $\begin{array}{l}\text { Existencia del ámbito de } \\
\text { planificación, explícito en el } \\
\text { comité directivo }\end{array}$ & $\begin{array}{l}\text { Variable dicotómica que toma el valor " } 1 \text { " cuando el proyecto } \\
\text { evidencia explícitamente que posee el ámbito de planificación en } \\
\text { el comité directivo y toma el valor "0" en caso contrario. }\end{array}$ \\
\hline $\begin{array}{l}\text { Planificación } \\
\text { reflejado en el } \\
\text { comité ejecutivo }\end{array}$ & $\begin{array}{l}\text { Existencia del ámbito de } \\
\text { planificación, explícito en el } \\
\text { comité ejecutivo }\end{array}$ & $\begin{array}{l}\text { Variable dicotómica que toma el valor " } 1 \text { " cuando el proyecto } \\
\text { evidencia explícitamente que posee el ámbito de planificación en } \\
\text { el comité ejecutivo y toma el valor "0" en caso contrario. }\end{array}$ \\
\hline $\begin{array}{l}\text { Aseguramiento de } \\
\text { la calidad } \\
\text { reflejado en el } \\
\text { Proyecto }\end{array}$ & $\begin{array}{l}\text { Existencia del ámbito de } \\
\text { aseguramiento de la } \\
\text { calidad, explícito en el } \\
\text { proyecto }\end{array}$ & $\begin{array}{l}\text { Variable dicotómica que toma el valor " } 1 \text { " cuando el proyecto } \\
\text { evidencia explícitamente que posee el ámbito de aseguramiento } \\
\text { de la calidad y toma el valor "0" en caso contrario. }\end{array}$ \\
\hline $\begin{array}{l}\text { Aseguramiento de } \\
\text { la calidad } \\
\text { reflejado en el } \\
\text { comité directivo }\end{array}$ & $\begin{array}{l}\text { Existencia del ámbito de } \\
\text { aseguramiento de la } \\
\text { calidad, explícito en el } \\
\text { comité directivo }\end{array}$ & $\begin{array}{l}\text { Variable dicotómica que toma el valor "1" cuando el proyecto } \\
\text { evidencia explícitamente que posee el ámbito de aseguramiento } \\
\text { de la calidad en el comité directivo y toma el valor "0" en caso } \\
\text { contrario. }\end{array}$ \\
\hline $\begin{array}{l}\text { Aseguramiento de } \\
\text { la calidad } \\
\text { reflejado en el } \\
\text { comité ejecutivo }\end{array}$ & $\begin{array}{l}\text { Existencia del ámbito de } \\
\text { aseguramiento de la } \\
\text { calidad, explícito en el } \\
\text { comité ejecutivo }\end{array}$ & $\begin{array}{l}\text { Variable dicotómica que toma el valor " } 1 \text { " cuando el proyecto } \\
\text { evidencia explícitamente que posee el ámbito de aseguramiento } \\
\text { de la calidad en el comité ejecutivo y toma el valor "0" en caso } \\
\text { contrario. }\end{array}$ \\
\hline
\end{tabular}




\section{RESULTADOS}

En la tabla 3 se muestra el resumen de los años y áreas de acreditación institucional de las universidades públicas en Chile, a partir de www.cnachile.cl, 25.09.2017. Como se puede observar en la tabla 3, el $100 \%$ de las universidades públicas se encuentran acreditadas. Además, en promedio los años de acreditación son 4,62 y las áreas son aproximadamente 4. Al realizar un análisis por área, se observa que es mayor el porcentaje del área de vinculación con el medio, 93.75\%, seguido de investigación, 62.50\% y postgrado, en un $25 \%$. En concreto, en este estudio se realiza una asociación directa cuando el concepto es explícito en el proyecto. Sin embargo, también se consideraron otros aspectos relacionados que fueron asociados a los conceptos estudiados y que se muestran en la tabla 4.

Tabla 3: Áreas y Años de Acreditación de las Universidades

\begin{tabular}{|l|c|}
\hline \multicolumn{1}{|c|}{ Aspecto } & Universidades Públicas \\
\hline Universidades acreditadas & $100 \%(16 / 16)$ \\
\hline Promedio de años acreditados & 4,62 \\
\hline Promedio de áreas acreditadas & 3,81 \\
\hline Promedio de años en Investigación & 5,10 \\
\hline Porcentaje de años en Investigación & $62,50 \%(10 / 16)$ \\
\hline Promedio de años en Postgrado & 5,75 \\
\hline Porcentaje de años en Postgrado & $25 \%(4 / 16)$ \\
\hline Promedio de años en Vinculación con el Medio & 4,73 \\
\hline Porcentaje de años en Vinculación con el Medio & $93,75 \%(15 / 16)$ \\
\hline
\end{tabular}

Tabla 4: Conceptos adicionales para la asociación con los ámbitos

\begin{tabular}{|c|c|c|c|}
\hline Ámbito & Área & Aspectos Considerados & Aspectos No Considerados \\
\hline \multirow{5}{*}{$\begin{array}{l}\text { Acreditación } \\
\text { Institucional }\end{array}$} & Docencia & $\begin{array}{l}\text { FID; Estudiantes; Modelo educativo } \\
\text { Capacitación académica } \\
\text { Aseguramiento de calidad de pregrado } \\
\text { Docencia pregrado } \\
\text { Acreditación del pregrado; Gestión académica }\end{array}$ & $\begin{array}{l}\text { Contratación de doctores } \\
\text { Oficina seguimiento de } \\
\text { egresado } \\
\text { Dpto. administración y } \\
\text { registro curricular }\end{array}$ \\
\hline & Investigación & $\begin{array}{l}\text { Vicerrector Investigación y Postgrado } \\
\text { Innovación; Innovación y transferencia tecnológica }\end{array}$ & \\
\hline & Postgrado & $\begin{array}{l}\text { Vicerrector Investigación y Postgrado } \\
\text { Programas de doctorados; Doctorantes } \\
\text { Concurso de postdoctorado, Contrato doctores } \\
\text { Fortalecer capacidades y competencias doctorantes }\end{array}$ & \\
\hline & Vinculación & $\begin{array}{l}\text { Director de vínculos } \\
\text { Relaciones institucionales, Vinculación con el Medio } \\
\text { Relaciones universitarias, Vinculación bidireccional } \\
\text { Director de movilidad asociado al cargo de VCM } \\
\text { Vicerrectoría TT y extensión con cargo en CM de VCM } \\
\text { Director de extensión y comunicación } \\
\text { Director de extensión y formación continua } \\
\text { Director cooperación internacional }\end{array}$ & $\begin{array}{l}\text { TT y extensión } \\
\text { Director TT y } \\
\text { comunicaciones } \\
\text { Innovación y TT } \\
\text { Director de TT }\end{array}$ \\
\hline & Gestión & $\begin{array}{l}\text { Fortalecimiento Institucional } \\
\text { Capacidades institucionales. } \\
\text { Planificación Estratégica, está asociada al área gestión, } \\
\text { pero gestión no se consideró como planificación. } \\
\text { Vicerrector de Asuntos Económicos (VRAE), Vicerrector } \\
\text { Adm. y Finanzas, Rector, Prorrector }\end{array}$ & \\
\hline \multirow{2}{*}{ Gestión } & $\begin{array}{l}\text { Planificación } \\
\text { Estratégica }\end{array}$ & $\begin{array}{l}\text { Plan Plurianual; Director de Análisis y Desarrollo } \\
\text { Institucional; Director de desarrollo estratégico } \\
\text { Vicerrector de Desarrollo }\end{array}$ & $\begin{array}{l}\text { Directora unidad proyectos } \\
\text { estratégicos }\end{array}$ \\
\hline & $\begin{array}{l}\text { Aseguramiento } \\
\text { de la calidad }\end{array}$ & $\begin{array}{l}\text { Sistema de gestión de calidad } \\
\text { Acreditación institucional; Mejoramiento continuo } \\
\text { Autoevaluación; Acreditación del pregrado }\end{array}$ & \\
\hline
\end{tabular}


Como se puede observar, en la figura 2 y con mayor detalle en la tabla 5 se muestran los tres proyectos, en los cuales se comprometen acciones principalmente asociadas al área de gestión e investigación, 100\%; seguido de vinculación, planificación y postgrado. Siendo menor el número de acciones vinculadas al área docencia y aseguramiento de la calidad. De manera complementaria, la tabla 6 muestra en detalle la información. En promedio, los proyectos convenio marco reflejan que las instituciones comprometen acciones en las 5 áreas aproximadamente, en un $93,75 \%$ se formulan objetivos asociados a planificación estratégica y en un $64,58 \%$ al aseguramiento de la calidad. La tabla 6 muestra la asignación presupuestaria por macrozona. Como se puede apreciar en la tabla 6 se muestra, la mayoría de los recursos se asignan en la zona central, $55,25 \%$ en promedio.

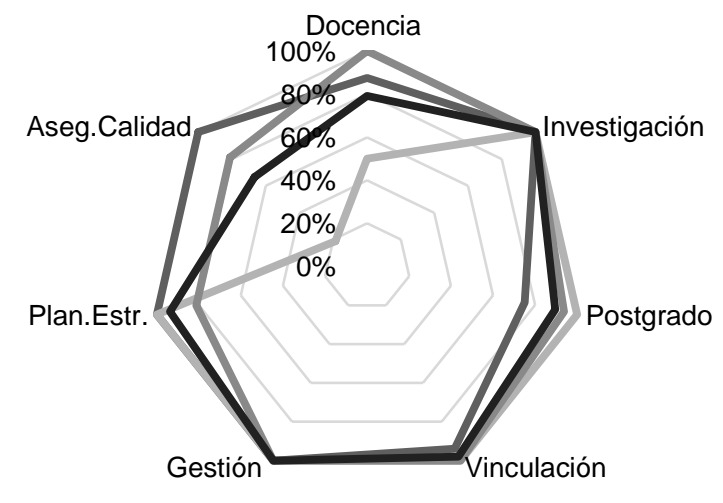

$\longrightarrow$ CM $2015 \longrightarrow$ CM 2016_1 CM 2016_2 Promedio

Fig. 2: Áreas de acreditación institucional, planificación estratégica y aseguramiento de la calidad reflejada en los proyectos (\%)

Tabla 5: Áreas de acreditación, planificación y aseguramiento de la calidad reflejadas en los proyectos

\begin{tabular}{|c|c|c|c|c|c|c|c|c|c|c|c|c|c|c|}
\hline \multirow{2}{*}{$\begin{array}{c}\text { Convenio } \\
\text { Marco }\end{array}$} & \multicolumn{2}{|c|}{$\begin{array}{c}\text { Áreas } \\
\text { Acreditación } \\
\text { Proyecto }\end{array}$} & \multicolumn{2}{|c|}{ Promedio } & \multirow{2}{*}{$\begin{array}{c}\text { Años de la } \\
\text { institución } \\
\text { Acred. Inst. } \\
№\end{array}$} & \multirow{2}{*}{$\begin{array}{c}\begin{array}{c}\text { Áreas de la } \\
\text { institución } \\
\text { Acred.Inst. }\end{array} \\
N^{\circ}\end{array}$} & \multicolumn{2}{|c|}{$\begin{array}{c}\text { Plan. } \\
\text { Estratégica }\end{array}$} & \multicolumn{2}{|c|}{ Promedio } & \multicolumn{2}{|c|}{$\begin{array}{l}\text { Aseg. } \\
\text { Calidad }\end{array}$} & \multicolumn{2}{|c|}{ Promedio } \\
\hline & № & $\%$ & № & $\%$ & & & № & $\%$ & № & $\%$ & № & $\%$ & № & $\%$ \\
\hline CM 2015 & 4,56 & 91 & \multirow{3}{*}{4,66} & \multirow{3}{*}{93,27} & 4,62 & 3,81 & 16 & 100 & \multirow{3}{*}{15} & \multirow{3}{*}{93,75} & 16 & 100 & \multirow{3}{*}{10,33} & \multirow{3}{*}{64,58} \\
\hline CM 2016_1 & 4,50 & 90 & & & 4,62 & 3,81 & 16 & 100 & & & 3 & 18,75 & & \\
\hline CM 2016_2 & 4,93 & 99 & & & 4,62 & 3,81 & 13 & 81 & & & 12 & 75 & & \\
\hline
\end{tabular}

Tabla 6: Asignación presupuestaria por macrozona de los Convenio Marco

\begin{tabular}{|c|c|c|c|c|c|c|c|c|c|c|}
\hline \multirow[t]{2}{*}{ Zona } & \multirow[t]{2}{*}{ Universidad } & \multirow[t]{2}{*}{ № } & \multirow[t]{2}{*}{ Región } & \multicolumn{4}{|c|}{ Recursos asignados al Convenio Marco } & \multicolumn{2}{|c|}{$\begin{array}{c}\text { Total por Zona } \\
\text { (\%) }\end{array}$} & \multirow{2}{*}{$\begin{array}{c}\text { Prome } \\
\text { dio } \\
\text { (\%) }\end{array}$} \\
\hline & & & & 2015 & $\%$ & 2016 & $\%$ & 2015 & 2016 & \\
\hline Norte & U. de Tarapacá & $\mathrm{XV}$ & $\begin{array}{c}\text { Arica y } \\
\text { Parinacota }\end{array}$ & 2.144 .629 & 6,29 & 2.815 .853 & 6,05 & \multirow{5}{*}{22,26} & \multirow{5}{*}{22,48} & \multirow{5}{*}{22,37} \\
\hline Norte & U. de Antofagasta & II & Antofagasta & 2.169 .063 & 6,36 & 2.374 .627 & 5,10 & & & \\
\hline Norte & U. Arturo Prat & 1 & Tarapacá & 821.677 & 2,41 & 2.248 .229 & 4,83 & & & \\
\hline Norte & U. de la Serena & IV & Coquimbo & 1.226 .300 & 3,60 & 1.736 .021 & 3,73 & & & \\
\hline Norte & U. de Atacama & III & Atacama & 1.223 .200 & 3,59 & 1.288 .544 & 2,77 & & & \\
\hline Centro & U. de Chile & XIII & Metropolitana & 5.609 .256 & $\begin{array}{c}16,4 \\
6\end{array}$ & 8.936 .068 & $\begin{array}{c}19,2 \\
0\end{array}$ & \multirow{4}{*}{54,98} & \multirow{4}{*}{55,51} & \multirow{4}{*}{55,25} \\
\hline Centro & U. de Santiago & XIII & Metropolitana & 3.059 .502 & 8,98 & 4.902 .887 & $\begin{array}{c}10,5 \\
3\end{array}$ & & & \\
\hline Centro & U. de Talca & VII & Maule & 3.193 .145 & 9,37 & 4.090 .748 & 8,79 & & & \\
\hline Centro & U. de Valparaíso & V & Valparaíso & 2.678 .770 & 7,86 & 2.761 .571 & 5,93 & & & \\
\hline
\end{tabular}


Tabla 6 (continuación)

\begin{tabular}{|c|c|c|c|c|c|c|c|c|c|c|}
\hline Centro & U. Tec. Metrop. & XIII & Metropolitana & 1.215 .376 & 3,57 & 2.181 .918 & 4,69 & & & \\
\hline Centro & U. de Playa Ancha & V & Valparaíso & 1.399 .646 & 4,11 & 1.513 .762 & 3,25 & & & \\
\hline Centro & $\begin{array}{l}\text { U. Metropolitana } \\
\text { de Cs de la Educ. }\end{array}$ & XIII & Metropolitana & 1.582 .414 & 4,64 & 1.446 .491 & 3,11 & & & \\
\hline Sur & U. del Bío- Bío & VIII & Biobío & 1.779 .283 & 5,22 & 2.662 .617 & 5,72 & \multirow{4}{*}{22,76} & \multirow{4}{*}{22,01} & \multirow{4}{*}{22,39} \\
\hline Sur & U. de la Frontera & IX & Araucanía & 3.109 .714 & 9,12 & 3.629 .363 & 7,80 & & & \\
\hline Sur & U. de Los Lagos & $X$ & Los Lagos & 1.458 .370 & 4,28 & 2.376 .223 & 5,11 & & & \\
\hline Sur & U. de Magallanes & XII & Magallanes & 1.409 .655 & 4,14 & 1.574 .671 & 3,38 & & & \\
\hline \multicolumn{4}{|c|}{ Total } & 34.080 .000 & 100 & 46.539 .593 & 100 & 100 & 100 & 100 \\
\hline
\end{tabular}

En la figura 3 se muestra si las áreas de acreditación institucional, planificación estratégica y aseguramiento de la calidad, se encuentran presentes en el comité directivo de los proyectos. En particular, se observa que en el $100 \%$ de los proyectos se refleja el área de gestión, seguido del área de docencia, investigación y postgrado. Además, menor es la presencia de cargos asociados a las áreas de planificación y vinculación. En cambio, no existen cargos asociados al área de aseguramiento de la calidad, en el comité directivo.

En cambio, en la tabla 7 se muestra evidencia los porcentajes de representación del Director y las áreas de acreditación, planificación y aseguramiento de la calidad, asociadas al comité directivo. Como se puede observar, en un $68,75 \%$ el director del proyecto es el Rector, seguido con un $12,50 \%$ de un Vicerrector o Director. Siendo el número de áreas de acreditación institucional, reflejados en el comité directivo, del convenio marco de aproximadamente 3 áreas, cuando el director del proyecto es Rector y Vicerrector. A la vez, se observa una mayor presencia de directivos asociados al cargo de planificación, 100\% cuando quien dirige el proyecto es un Prorrector(a), seguido de un $89 \%$ cuando es una dirección. A diferencia de aseguramiento de la calidad, cuyo porcentaje mayor es de un $16,67 \%$, cuando quién dirige es un VRAE.

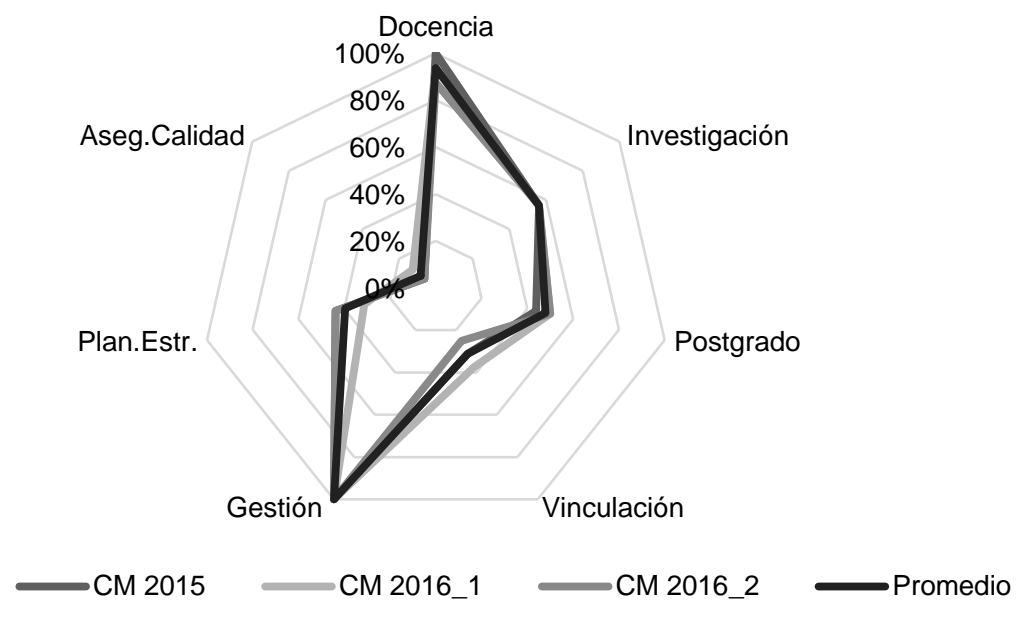

Fig. 3: Áreas de acreditación institucional, planificación estratégica y aseguramiento de la calidad reflejadas en el comité directivo

En la figura 4 se muestra si las áreas de acreditación institucional, planificación estratégica y aseguramiento de la calidad, se encuentran presentes en el comité ejecutivo de los proyectos. Se observa que principalmente en los proyectos se refleja el área de gestión, seguido del área de investigación, VCM, docencia y postgrado. Además, menor es la presencia de cargos asociados a las áreas de planificación y aseguramiento de la calidad. En la tabla 8 se muestra con mayor detalle los porcentajes de representación del director alterno y las áreas de acreditación, planificación y aseguramiento de la calidad, asociadas al comité ejecutivo. Para mayor análisis se desagrega la categoría vicerrector, y, además, se asocia el cargo de vicerrector de campus, a la categoría de Prorrector(a), porque este cargo involucra diferentes ámbitos, que abordan las categorías de vicerrectores, tales como: Vicerrector Académico (VRA), VRAE, entre otros. 
Tabla 7: Porcentaje de representación del Director y áreas de acreditación, planificación y aseguramiento de la calidad, asociadas al comité directivo

\begin{tabular}{|c|c|c|c|c|c|c|c|c|c|c|c|c|c|c|c|}
\hline \multirow[t]{2}{*}{$\begin{array}{l}\text { Convenio } \\
\text { Marco }\end{array}$} & \multirow[t]{2}{*}{$\begin{array}{l}\text { Director } \\
\text { Principal }\end{array}$} & \multirow{2}{*}{$\begin{array}{c}\% \\
\text { promedio } \\
d e \\
\text { director } \\
\text { principal }\end{array}$} & \multirow{2}{*}{$\begin{array}{c}\% \\
\text { promedio } \\
\text { de } \\
\text { director } \\
\text { principal }\end{array}$} & \multicolumn{2}{|c|}{$\begin{array}{c}\text { Áreas } \\
\text { Acreditación } \\
\text { Equipo } \\
\text { Directivo }\end{array}$} & \multicolumn{2}{|c|}{ Promedio } & \multicolumn{2}{|c|}{$\begin{array}{c}\text { Plan. } \\
\text { Estratégica }\end{array}$} & \multicolumn{2}{|c|}{ Promedio } & \multicolumn{2}{|c|}{$\begin{array}{l}\text { Aseg. } \\
\text { Calidad }\end{array}$} & \multicolumn{2}{|c|}{ Promedio } \\
\hline & & & & № & $\%$ & № & $\%$ & № & $\%$ & № & $\%$ & № & $\%$ & № & $\%$ \\
\hline 2015 & \multirow{3}{*}{ Rector } & 68,75 & \multirow{3}{*}{68,75} & 3,5 & 71 & \multirow{3}{*}{3,55} & \multirow{3}{*}{71} & 4,0 & 36 & \multirow{3}{*}{3,00} & \multirow{3}{*}{28} & 1 & 9,09 & \multirow{3}{*}{1,0} & \multirow{3}{*}{9,14} \\
\hline 2016_1 & & 75,00 & & 3,4 & 68 & & & 1,0 & 8 & & & 1 & 8,33 & & \\
\hline 2016_2 & & 62,50 & & 3,7 & 74 & & & 4,0 & 40 & & & 1 & 10 & & \\
\hline 2015 & \multirow{3}{*}{ Prorrector } & 6,25 & \multirow{3}{*}{6,25} & 3,0 & 60 & \multirow{3}{*}{1,67} & \multirow{3}{*}{33} & 1,0 & 100 & \multirow{3}{*}{1,00} & \multirow{3}{*}{100} & 0 & 0 & \multirow{3}{*}{0,0} & \multirow{3}{*}{0} \\
\hline 2016_1 & & 6,25 & & 1,0 & 20 & & & 1,0 & 100 & & & 0 & 0 & & \\
\hline 2016_2 & & 6,25 & & 1,0 & 20 & & & 1,0 & 100 & & & 0 & 0 & & \\
\hline 2015 & \multirow{3}{*}{ VRAE } & 6,25 & \multirow{3}{*}{12,50} & 4,0 & 80 & \multirow{3}{*}{3,87} & \multirow{3}{*}{78} & 0,0 & 0 & \multirow{3}{*}{0,67} & \multirow{3}{*}{33} & 0 & 0 & \multirow{3}{*}{0,3} & \multirow{3}{*}{16,67} \\
\hline 2016_1 & & 12,50 & & 5,0 & 100 & & & 2,0 & 100 & & & 1 & 50 & & \\
\hline 2016_2 & & 18,75 & & 2,6 & 53 & & & 0,0 & 0 & & & 0 & 0 & & \\
\hline 2015 & & 18,75 & & 2,3 & 47 & & & 2,0 & 67 & & & 0 & 0 & & \\
\hline 2016_1 & Director & 6,25 & 12,50 & 2,0 & 40 & 2,27 & 46 & 1,0 & 100 & 1,67 & 89 & 0 & 0 & 0,0 & 0 \\
\hline $2016 \_2$ & & 12,50 & & 2,5 & 50 & & & 2,0 & 100 & & & 0 & 0 & & \\
\hline
\end{tabular}

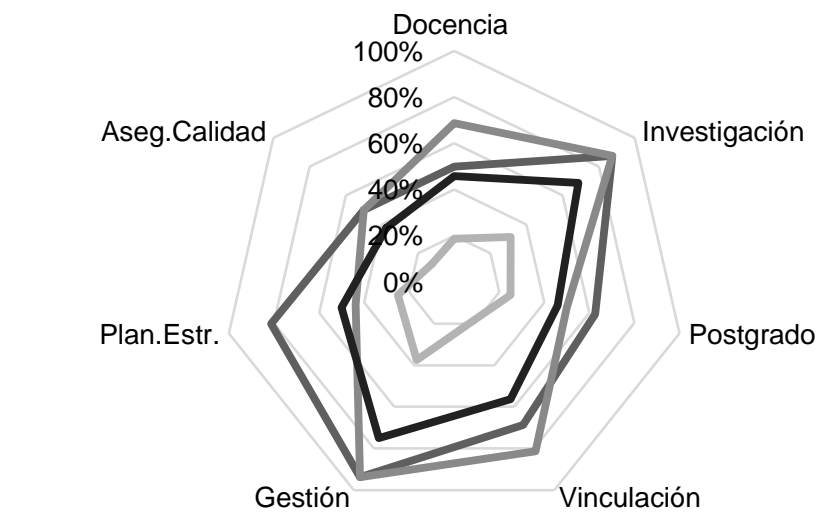

$\longrightarrow$ CM $2015 \longrightarrow$ CM 2016_1 C

Fig. 4: Áreas de acreditación institucional, planificación estratégica y aseguramiento de la calidad reflejadas en el comité ejecutivo

Tabla 8: Resumen de la dependencia de los proyectos Convenio Marco (\%)

\begin{tabular}{|c|c|c|c|c|c|c|c|c|}
\hline \multirow{2}{*}{ Cargo } & \multicolumn{4}{|c|}{ Director } & \multicolumn{4}{|c|}{ Director Alterno } \\
\hline & $\begin{array}{c}\text { CM } \\
2015 \\
\end{array}$ & $\begin{array}{c}\text { CM } \\
2016 \_1\end{array}$ & $\begin{array}{c}\text { CM } \\
2016 \_2 \\
\end{array}$ & $\begin{array}{c}\% \text { total } \\
\text { (proyectos) }\end{array}$ & $\begin{array}{c}\text { CM } \\
2015 \\
\end{array}$ & $\begin{array}{c}\text { CM } \\
2016 \_1 \\
\end{array}$ & $\begin{array}{c}\text { CM } \\
2016 \_2\end{array}$ & $\begin{array}{c}\% \text { total } \\
\text { (proyectos) }\end{array}$ \\
\hline Rector & $68,75 \%$ & $75,00 \%$ & $62,50 \%$ & $\begin{array}{l}(33 / 48) \\
68,75 \% \\
\end{array}$ & $6,25 \%$ & $6,25 \%$ & $6,25 \%$ & $\begin{array}{l}(3 / 48) \\
6,25 \% \\
\end{array}$ \\
\hline Prorrector(a) & $6,25 \%$ & $6,25 \%$ & $6,25 \%$ & $\begin{array}{l}(3 / 48) \\
6,25 \% \\
\end{array}$ & $\begin{array}{c}18,75 \\
\%\end{array}$ & $18,75 \%$ & $18,75 \%$ & $\begin{array}{l}(11 / 48) \\
22,92 \% \\
\end{array}$ \\
\hline $\begin{array}{l}\text { Vicerrectoría } \\
\mathrm{s}\end{array}$ & $6,25 \%$ & $12,50 \%$ & $18,75 \%$ & $\begin{array}{l}(6 / 48) \\
12,5 \% \\
\end{array}$ & $\begin{array}{c}68,75 \\
\% \\
\end{array}$ & $62,50 \%$ & $68,75 \%$ & $\begin{array}{l}(30 / 48) \\
62,50 \% \\
\end{array}$ \\
\hline Director & $18,75 \%$ & $6,25 \%$ & $12,50 \%$ & $\begin{array}{l}(6 / 48) \\
12,5 \% \\
\end{array}$ & $6,25 \%$ & $12,50 \%$ & $6,25 \%$ & $\begin{array}{l}(4 / 48) \\
8,33 \% \\
\end{array}$ \\
\hline TOTAL & $100 \%$ & $100 \%$ & $100 \%$ & $100 \%$ & $100 \%$ & $100 \%$ & $100 \%$ & $100 \%$ \\
\hline
\end{tabular}

En la tabla 8 se muestra el resumen de la dependencia de los convenios marco, en donde se observa que es menor el porcentaje de dirección del proyecto, cuando se es Prorrector(a), y de ser director alterno, cuando 
se es Rector o Director. Específicamente, la tabla 9 muestra que el principal director alterno de los convenios marco es el vicerrector académico, con un $39,58 \%$, seguido del vicerrector económico, con un $20,83 \%$. Además, al agrupar por Vicerrector se aprecia que el $62.50 \%$, es la categoría que principalmente se asocia como director alterno. Además, como se puede apreciar en la tabla 10 se muestra, el cargo de planificación se encuentra presente en un $50 \%$ en el comité ejecutivo y un $40 \%$ en el comité directivo. En cambio, menor es el porcentaje asociado al cargo de aseguramiento de la calidad en ambos comités, $38 \%$ y $8 \%$, respectivamente.

Tabla 9: Porcentaje de representación del Director alterno y áreas de acreditación, planificación y aseguramiento de la calidad, asociadas al comité ejecutivo

\begin{tabular}{|c|c|c|c|c|c|c|c|c|c|c|c|c|c|c|c|}
\hline \multirow[t]{2}{*}{$\begin{array}{c}\text { Convenio } \\
\text { Marco }\end{array}$} & \multirow[t]{2}{*}{$\begin{array}{l}\text { Director } \\
\text { alterno }\end{array}$} & \multirow{2}{*}{$\begin{array}{c}\% \\
\text { acumulad } \\
\text { o de } \\
\text { director } \\
\text { alterno } \\
\text { (por CM) }\end{array}$} & \multirow{2}{*}{$\begin{array}{c}\% \\
\text { acumulado } \\
\text { promedio } \\
\text { de director } \\
\text { alterno }\end{array}$} & \multicolumn{2}{|c|}{$\begin{array}{c}\text { Áreas } \\
\text { Acreditaci } \\
\text { ón Equipo } \\
\text { Ejecutivo }\end{array}$} & \multicolumn{2}{|c|}{ Prom. } & \multicolumn{2}{|c|}{$\begin{array}{c}\text { Plan. } \\
\text { Estratégica }\end{array}$} & \multicolumn{2}{|c|}{ Prom. } & \multicolumn{2}{|c|}{$\begin{array}{l}\text { Aseg. } \\
\text { Calidad }\end{array}$} & \multicolumn{2}{|c|}{ Prom. } \\
\hline & & & & № & $\%$ & № & $\%$ & № & $\%$ & № & $\%$ & № & $\%$ & № & $\%$ \\
\hline 2015 & \multirow{3}{*}{ Rector } & 6,25 & \multirow{3}{*}{6,25} & 5,0 & $\begin{array}{c}10 \\
0\end{array}$ & \multirow{3}{*}{3,0} & \multirow{3}{*}{60} & 1,0 & 100 & \multirow{3}{*}{0,6} & \multirow{3}{*}{67} & 1 & $\begin{array}{c}10 \\
0\end{array}$ & \multirow{3}{*}{0,6} & \multirow{3}{*}{67} \\
\hline 2016_1 & & 6,25 & & 0,0 & 0 & & & 0,0 & 0 & & & 0 & 0 & & \\
\hline 2016_2 & & 6,25 & & 4,0 & 80 & & & 1,0 & 100 & & & 1 & $\begin{array}{c}10 \\
0\end{array}$ & & \\
\hline 2015 & \multirow{3}{*}{$\begin{array}{c}\text { Prorrector y } \\
\text { Vicerrector } \\
\text { Campus }\end{array}$} & 25,00 & \multirow{3}{*}{22,92} & 3,5 & 70 & \multirow{3}{*}{2,5} & \multirow{3}{*}{50} & 4,0 & 100 & \multirow{3}{*}{2,0} & \multirow{3}{*}{53} & 3 & 75 & \multirow{3}{*}{2,0} & \multirow{3}{*}{56} \\
\hline 2016_1 & & 25,00 & & 1,0 & 20 & & & 1,0 & 25 & & & 1 & 25 & & \\
\hline 2016_2 & & 18,75 & & 3,0 & 60 & & & 1,0 & 33 & & & 2 & 67 & & \\
\hline 2015 & \multirow{3}{*}{$\begin{array}{l}\text { Vicerrectoría } \\
\text { Investigación } \\
\text { y Postgrado }\end{array}$} & 0,00 & \multirow{3}{*}{2,08} & 0,0 & 0 & \multirow{3}{*}{1,0} & \multirow{3}{*}{20} & 0,0 & 0 & & & 0 & 0 & & \\
\hline 2016_1 & & 0,00 & & 0,0 & 0 & & & 0,0 & 0 & 0,3 & 33 & 0 & 0 & 0,0 & 0 \\
\hline 2016_2 & & 6,25 & & 3,0 & 60 & & & 1,0 & 100 & & & 0 & 0 & & \\
\hline 2015 & & 37,50 & & 3,6 & 73 & & & 3,0 & 50 & & & 3 & 50 & & \\
\hline 2016_1 & VRA & 37,50 & 39,58 & 1,5 & 30 & 3,1 & 63 & 1,0 & 17 & 2,3 & 37 & 1 & 17 & 2,3 & 37 \\
\hline 2016_2 & & 43,75 & & 4,2 & 86 & & & 3,0 & 43 & & & 3 & 43 & & \\
\hline 2015 & & 25,00 & & 3,5 & 70 & & & 4,0 & 100 & & & 1 & 25 & & \\
\hline 2016_1 & VRAE & 18,75 & 20,83 & 1,6 & 33 & 2,9 & 59 & 1,0 & 33 & 2,0 & 56 & 0 & 0 & 0,6 & 19 \\
\hline 2016_2 & & 18,75 & & 3,6 & 73 & & & 1,0 & 33 & & & 1 & 33 & & \\
\hline 2015 & & 6,25 & & 3,0 & 60 & & & 1,0 & 100 & & & 0 & 0 & & \\
\hline 2016_1 & Director & 12,50 & 8,33 & 1,5 & 30 & 2,8 & 57 & 1,0 & 50 & 0,6 & 50 & 0 & 0 & 0,3 & 33 \\
\hline 2016_2 & & 6,25 & & 4,0 & 80 & & & 0,0 & 0 & & & 1 & $\begin{array}{c}10 \\
0\end{array}$ & & \\
\hline 2015 & & 62,50 & & 3,6 & 72 & & & 7,0 & 70 & & & 4 & 40 & & \\
\hline 2016_1 & $\begin{array}{l}\text { Vicerrectorías } \\
\text { (aqrupado) }\end{array}$ & 56,25 & 62,50 & 1,5 & 31 & 3,0 & 61 & 2,0 & 22 & 4,6 & 46 & 1 & 11 & 3,0 & 29 \\
\hline 2016_2 & & 68,75 & & 4,0 & 80 & & & 5,0 & 45 & & & 4 & 36 & & \\
\hline
\end{tabular}

Tabla 10: Resumen de la presencia de planificación y aseguramiento de la calidad en los proyectos Convenio Marco (\%)

\begin{tabular}{|c|c|c|c|c|c|c|c|c|c|}
\hline \multirow{2}{*}{$\begin{array}{c}\text { Convenio } \\
\text { Marco }\end{array}$} & \multirow{2}{*}{ Comité } & \multicolumn{2}{|c|}{ Plan. Estratégica } & \multicolumn{2}{|c|}{ Promedio } & \multicolumn{2}{|c|}{ Aseg. Calidad } & \multicolumn{2}{|c|}{ Promedio } \\
\hline & & $N^{o}$ & $\%$ & № & $\%$ & $N^{\circ}$ & $\%$ & $N^{\circ}$ & $\%$ \\
\hline CM 2015 & \multirow{3}{*}{ Directivo } & 7,0 & 44 & \multirow{3}{*}{6,33} & \multirow{3}{*}{40} & 1,0 & 6 & \multirow{3}{*}{1,33} & \multirow{3}{*}{8} \\
\hline CM 2016_1 & & 5,0 & 31 & & & 2,0 & 13 & & \\
\hline CM 2016_2 & & 7,0 & 44 & & & 1,0 & 6 & & \\
\hline CM 2015 & \multirow{3}{*}{ Ejecutivo } & 13,0 & 81 & \multirow{3}{*}{8,00} & \multirow{3}{*}{50} & 8,0 & 50 & \multirow{3}{*}{6,00} & \multirow{3}{*}{38} \\
\hline CM 2016_1 & & 4,0 & 25 & & & 2,0 & 13 & & \\
\hline CM 20162 & & 7,0 & 44 & & & 8,0 & 50 & & \\
\hline
\end{tabular}

Finalmente, los proyectos convenio marco involucran objetivos en red, y aunque no es el objetivo de análisis de este estudio, de manera resumida la tabla 11 muestra la asociación de áreas de acreditación y ámbitos de 
las redes constituidas en los convenios marco. Además, existe una red de coordinadores institucionales, que colaboran en la coordinación de las actividades que realizan.

Tabla 11: Relación entre las redes y las áreas de acreditación institucional

\begin{tabular}{|l|c|c|c|c|c|c|c|}
\hline \multirow{2}{*}{\multicolumn{1}{c|}{ Red }} & \multicolumn{3}{c|}{ Áreas de Acreditación Institucional } & \multicolumn{2}{c|}{ Ámbitos } \\
\cline { 2 - 8 } & Docencia & Investig. & Postg. & Vinculación & Gestión & Plan.Estrat. & Aseg.Calidad \\
\hline Gestión Econ. y Adm. & & & & & $\mathrm{x}$ & & \\
\hline Docencia de Pregrado & $\mathrm{x}$ & & & & & & \\
\hline Aseguramiento de la calidad & & & & & & & \\
\hline Vicerrectores de Investig. & & $\mathrm{x}$ & & & & & \\
\hline Arte, Cultura y Patrimonio & & & & $\mathrm{x}$ & & & \\
\hline Vinculación con el Medio & & & & $\mathrm{x}$ & & & \\
\hline Análisis Institucional & & & & & $\mathrm{x}$ & & \\
\hline
\end{tabular}

\section{Análisis de correspondencias múltiples}

En esta sección se presentan los resultados de un análisis de correspondencias múltiples, la cual es una técnica descriptiva que se utiliza para representar gráficamente una tabla de contingencia para más de dos variables categóricas. El objetivo consiste en analizar las variables del estudio a la vez. A continuación, la tabla 12 muestra las medidas discriminantes del análisis de correspondencias múltiples de las variables.

Tabla 12: Medidas discriminantes del análisis de correspondencias múltiples

\begin{tabular}{|l|c|c|c|}
\hline \multicolumn{1}{|c|}{ Variables } & \multicolumn{2}{c|}{ Dimensión } & \multirow{2}{*}{ Media } \\
\cline { 2 - 4 } & 1 & 2 & 0,617 \\
\hline Áreas Acreditación Institucional & 0,824 & 0,411 & 0,484 \\
\hline Años Acreditación Institucional & 0,791 & 0,178 & 0,153 \\
\hline$N^{\circ}$ áreas reflejadas en el proyecto & 0,190 & 0,115 & 0,615 \\
\hline Tipo de Director & 0,606 & 0,624 & 0,838 \\
\hline Tipo Director Alterno & 0,812 & 0,864 & 2,708 \\
\hline Total activo & 3,223 & 2,192 & 54,154 \\
\hline \% de varianza & 64,462 & 43,845 & \\
\hline
\end{tabular}

Como se puede observar la tabla 12 muestra el porcentaje de varianza explicada del modelo es de 54\%.

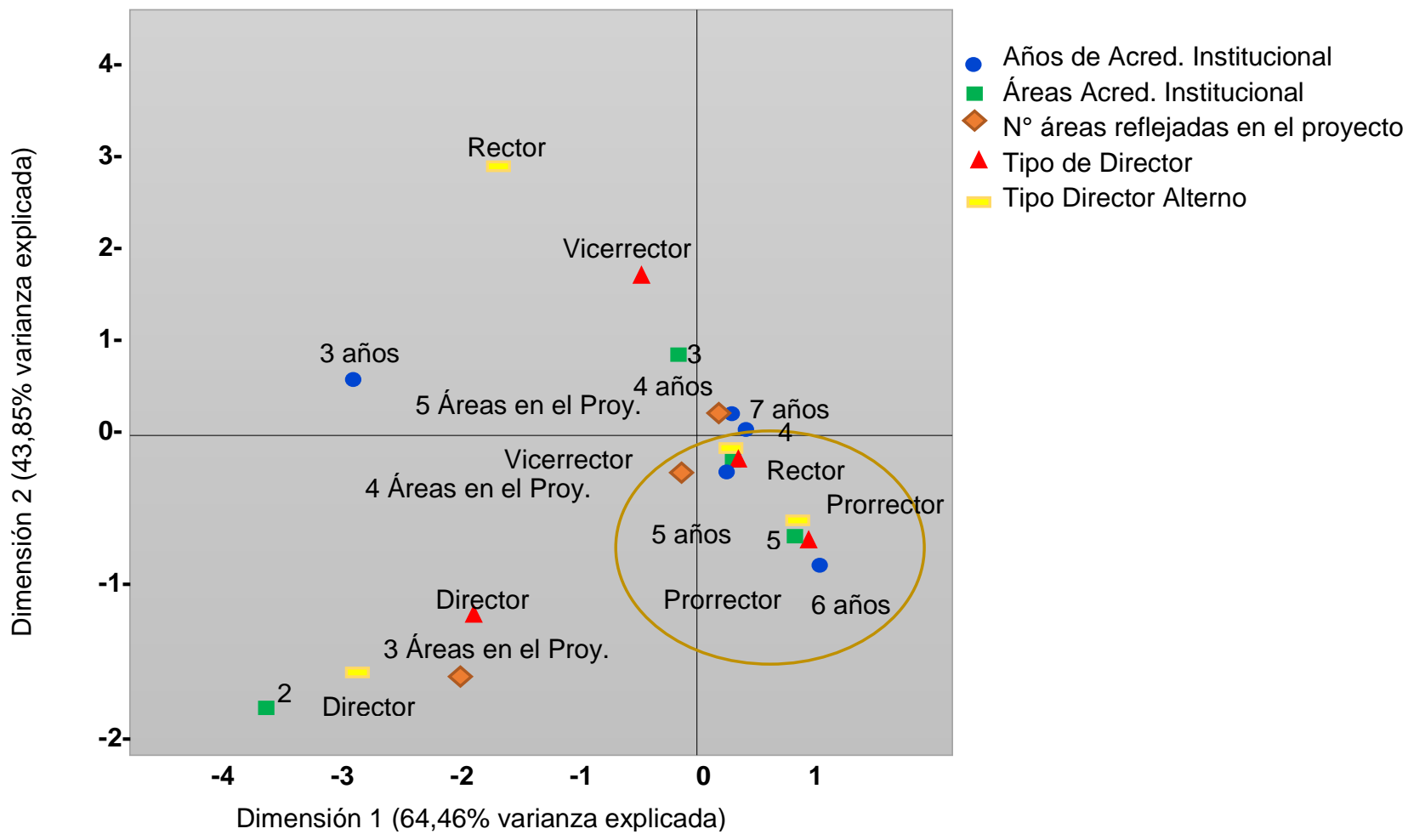

Fig. 5: Gráfico conjunto de puntos de categoría. Normalización principal por variable. 
En la figura 5 se muestra el diagrama conjunto de puntos de categorías de los proyectos convenio marco, donde la dimensión 1 presenta un porcentaje de varianza explicada de $64,46 \%$, donde la variable que se representa con mayor fuerza en esta dimensión, son los años y áreas de acreditación, a diferencia de la dimensión 2, que se encuentra más asociada al tipo de director del proyecto y tipo de director alterno. En concreto, como se puede observar, en el cuadrante IV, existe asociación entre las áreas y años de acreditación institucional, con el número de áreas reflejadas en los proyectos convenio marco, el tipo de director y el tipo de director alterno, que dirige los proyectos estratégicos convenio marco. A diferencia el cuadrante II y III, en los cuales no se percibe esta asociación.

\section{DISCUSIÓN}

Los resultados del estudio permiten apreciar que es menor el número de acciones vinculadas al área de docencia y aseguramiento de la calidad. Lo anterior, se encuentra en línea con lo planteado por Palma y Rodríguez (2017: 195), quienes señalan que la única labor docente sobre la que se focalizan los convenios marco, está constituida por la formación inicial de profesores. Además, se descubrió que los proyectos convenio marco reflejan que las instituciones comprometen acciones en las 5 áreas de la acreditación institucional, en un $94 \%$ se formulan objetivos asociados a planificación estratégica y en un $65 \%$ al aseguramiento de la calidad. No obstante, es menor el porcentaje de directivos cuyo cargo está asociado al área de planificación en el comité ejecutivo, $50 \%$, siendo de un $40 \%$ en el comité directivo, en cambio mucho menor es el número de directivos cuyo cargo representa el aseguramiento de la calidad en dichos comités, del $38 \%$ y $8 \%$, respectivamente. Por otra parte, aunque existe una diversidad de redes, se observa que ésta es limitada, reflejando la focalización de la cooperación entre universidades, tal como el estudio de Marques et al. (2016) en donde la cooperación entre universidades se orienta a fomentar el desarrollo tecnológico. Sin embargo, no existen redes de postgrado ni de planificación estratégica, pese a que el postgrado fomenta el desarrollo de capital humano avanzado y la planificación estratégica es el instrumento que guía a las instituciones, según Cuesta (2012), y es sobre la cual se basan los proyectos convenio marco.

Finalmente, este tipo de proyectos estratégicos en su mayoría los lidera el Rector, como Director del proyecto, y cuyo vicerrector académico cumple la función de director alterno. Lo cual es consistente con los argumentos previos, al plantear que los proyectos estratégicos deben estar asociados a altos cargos jerárquicos, para permitir la instalación y transversalidad en la institución. No obstante, este esquema trae consigo mayor carga de trabajo a los rectores, quienes, a su vez, son responsables de otras funciones dentro y fuera de la institución. Debido a que no existen mecanismos de cooperación similares en otros países, no se puede realizar un análisis comparativo, demostrando que esta forma de colaboración entre universidades públicas es pionera en Chile, siendo lo más cercano los proyectos que el Banco Mundial lleva adelante en diferentes países (Post et al., 2004: 213), o los estudios que relacionan el ámbito de planificación estratégica con proyectos (Amedzro et al., 2017; Stretton, 2018).

\section{CONCLUSIONES}

De acuerdo a los resultados del estudio, de su discusión y análisis se pueden extraer las siguientes tres conclusiones principales: 1) El convenio marco es un mecanismo de financiamiento único en el mundo que fomenta la cooperación entre universidades públicas; 2) En definitiva, de acuerdo a nuestra hipótesis bajo estudio, es posible demostrar que los convenios marco contribuyen, principalmente, al fortalecimiento de las áreas de acreditación institucional, al igual que, a la planificación estratégica. No así tanto al aseguramiento de la calidad, debido a que este concepto estaba menos explícito en los proyectos. No obstante, hay que considerar que estos tres conceptos están relacionados y se complementan dentro de la institución, lo cual se evidencia en el análisis de correspondencias múltiples que demostró la asociación entre ellos; 3) Los impactos del convenio marco deben ser estudiados a largo plazo, para verificar la permanencia del mejoramiento de la calidad sobre las instituciones de educación superior en el tiempo. Por otra parte, los convenios marco exigen una rendición mensual de los gastos e informes de avances para dar cuenta de los recursos públicos, lo cual demanda de parte de las universidades una gran responsabilidad social, propio de la naturaleza jurídica de una institución del Estado. Lo anterior, es necesario debido a que los recursos son escasos y por ello deben estar orientados en función de un proyecto estratégico, que busque la calidad y la excelencia. Por ello es importante destacar que estos proyectos son formulados por la propia institución, de acuerdo a su planificación estratégica y los desafíos institucionales que cada una persiga.

La consideración de estos elementos trae consigo diversas implicancias para la educación superior, tales como el impacto en los modelos educativos, al considerar los proyectos convenios marco desde una mirada estratégica, tanto en su formulación como en su ejecución, direccionando lo comprometido en ellos hacia áreas que sean de relevancia institucional, con la finalidad de fomentar el desarrollo universitario en beneficio de la comunidad tanto interna como externa. Asimismo, permite dirigir los recursos públicos hacia áreas de desarrollo estratégico, tanto nacional como internacional, debido a que deben estar orientados hacia aquellos 
ámbitos en los cuales se requiera una mayor focalización, para poder contribuir al crecimiento y desarrollo universitario. En definitiva, los proyectos convenio marco modifican la lógica de actuación del sistema de educación superior chileno, a partir de proyectos alineados a la planificación institucional, a las prioridades ministeriales, al igual que al trabajo en red entre universidades públicas, cambiando la forma de trabajo habitual en el sistema, buscando principalmente la cooperación y articulación entre las instituciones.

\section{REFERENCIAS}

Acosta, A.O., La FIMPES y la Mejora de la Calidad de Instituciones Privadas: Un Estudio Acerca del Concepto de Calidad y de los Procesos de Acreditación en Tres Universidades Particulares, ISSN: 01852760, Rev. de la Educ. Superior, 44(175), 169-175 (2015)

Amedzro, W., É. Agba y D. Dominic, Profitability During the Projects Administration: Strategic Change Determinants for Reconfiguring Project Planning, ISSN: 2371-4794, Management \& Applied Economics Review, 24, 15-26 (2017)

Araneda-Guirriman, C.A., J. Gairín-Sallán y L.M. Pedraja-Rejas, La Autonomía en la Educación Superior: Reflexiones desde los Actores en el Contexto del Financiamiento por Desempeño en Chile, doi: 10.4067/S0718-50062018000400065, Form. Univ. 11(4), 65-74 (2018)

Araneda-Guirriman, C.A. y L.M. Pedraja-Rejas, Financiamiento por Desempeño en Chile: Análisis Conceptual de un Instrumento para la Educación Superior, doi: 10.4067/S0718-50062016000300009, Form. Univ. 9(3), 75-86 (2016)

Cancino, V.E. y R. Schmal, Sistema de Acreditación Universitaria en Chile: ¿Cuánto Hemos Avanzado?, doi: 10.4067/S0718-07052014000100003, Estud. Pedagóg., XL (1), 41-60 (2014)

Casani, F., D. De Filippo y otros dos autores, Public Versus Private Universities: Assessment of Research Performance; Case Study of the Spanish University System, doi:10.1093/reseval/rvt028, Research Evaluation, 23(1), 48-61 (2014)

Cuesta, U., Planificación Estratégica y Creatividad, 35-42, ESIC Editorial, Madrid, España (2012)

De Francisco, M.V., M.T. Jiménez y otros dos autores, Evaluating Impacts of University Cooperation for Development from The Voice of the South, ISSN: 1576-0162, Revista de Economía Mundial, (47), 95-116 (2017)

García, J.F. y M. Henry, University Co-operation between Europe and Latin America, ISSN: 1465-3435, European Journal of Education, 28(1), 85-98 (1993)

Gómez, V.G, M.R. Tolozano y N.B. Delgado, La Acreditación Institucional de la Calidad en los Institutos Superiores Técnicos y Tecnológicos del Ecuador desde la Perspectiva de un Instituto Acreditado, doi:10.4067/S071850062017000600007, Form. Univ. 10(6), 59-66 (2017)

Kapliienko, M., International Cooperation of Izmail State University for Humanities in 2015-2016, ISSN: 2069-9344, EIRP Proceedings, 11, 444-447 (2016)

Keller, T., The Benefits of Cooperation between Industry and Universities, ISSN: 1805-4862, Central European Business Review, 1 (2), 58-58 (2012)

Leal, F.G., R.R. Céspedes y L. Stallivieri, The Profile of the International Cooperation University Manager in Brazil, doi: 10.18568/1980-4865.1221-16, Revista Electrônica de Negócios Internacionais da ESPM, 12 (2), 1-16 (2017)

Lukac, D. y M.M. Chatzimichailidou, Common Sense Approach as a Basis for Successful University-Industry Cooperation, ISSN: 2067 - 3809, Acta Technica Corvininesis - Bulletin of Engineering, 10(1), 84-89 (2017)

Marques, H.R., M. De Oliveira y otros dos autores, Cooperation For Technological Development: An Analysis In The Context Of Federal Universities of Minas Gerais State, doi.org/10.1016/j.rai.2016.02.003, Revista de Administração e Inovação - RAI, 13(1), 127-146 (2016)

Martínez, J.E., S. Tobon y A. Romero, Problemáticas Relacionadas con la Acreditación de la Calidad de la Educación Superior en América Latina, doi:1665-2673-ie-17-73-00079, Innovación Educativa, 17 (73), $79-96$ (2017)

Mollis, M., Administrar la Crisis de la Educación Pública y Evaluar la Calidad Universitaria en América Latina: Dos Caras de la Misma Reforma Educativa, doi.org/10.1016/j.resu.2014.01.001, Rev. de la Educ. Superior, 43 (169), 25-45 (2014)

Palma, Á.A. y E.R. Rodríguez, La Asignación de Recursos a las Universidades del Estado en un Contexto de Rendición de Cuenta: La Lógica de los Convenios Marco en el Caso de Chile, doi: 10.4067/S0718-33052017000200192, Ingeniare, 25(1) ,194-195 (2017)

Pavlin, S., Considering University-Business Cooperation Modes from the Perspective of Enterprises, doi: 10.1111/ejed.12163, European Journal of Education, 51(1), 25-39 (2016)

Pavlin, S., T. Kesting y T. Baaken, An Integrative View on Higher Education and University-Business Cooperation in the Light of Academic Entrepreneurship, doi: 10.1111/ejed.12168, European Journal of Education, 51(1), 3-9 (2016)

Post, D., L. Clipper y otros cuatro autores, World Bank Okays Public Interest in Higher Education, doi: 10.1023/B:HIGH.0000034315.98795.c8, Higher Education, 48(2), 213-229 (2004)

Ranga, M., J. Perälampi y J. Kansikas, The New Face of University-Business Cooperation in Finland, doi: 10.1093/scipol/scw044, Science \& Public Policy (SPP), 43(5), 601-612 (2016) 
Rõigas, K., P. Mohnen y U. Varblane, Which Firms Use Universities as Cooperation Partners? A Comparative View in Europe, doi:10.1504/IJTM.2018.10009595, International Journal of Technology Management, 76 (1/2), $32-57$ (2018)

Rossano, S., A. Meerman y otros dos autores, The Relevance of Problem-based Learning for Policy Development in University-Business Cooperation, doi: 10.1111/ejed.12165, European Journal of Education, 51 (1), $40-55$ (2016)

Spodarczyk, E. y K. Szelągowska, Is Social Responsibility Required in the Cooperation Among Universities, Businesses and Local Government in the Local Environment?, ISSN: 20489021, Proceedings of the European Conference on Management, Leadership \& Governance, 437-444 (2015)

Stretton, A., Relating Causes of Project Failure to an Organizational Strategic Business Framework, ISSN: 2330-4480, PM World Journal, 7(1), 1-10 (2018)

Thamae, T.M., R.I. Thamae y L.Z. Thamae, A Process Model for University-Industry Cooperation in Sub-Saharan Africa: Lessons from Lesotho, ISSN: 1750-4562, African Journal of Business \& Economic Research, 11 (2/3), 103-125 (2016)

Venables, J.P. y J. Van Gastel, Radiografía de los Modelos de Acreditación: Organización, Procesos y Prácticas. El Caso de las Universidades Austral de Chile, de la Frontera y de los Lagos, ISSN: 0718-4565, Calidad en la Educación, (41), 5181 (2014)

Yang, L., Province/Ministry- Coordinated Industry-University-Institute Cooperation and University Development: Based on the Experiences of Guangdong Province, doi: 10.1080/10611932.2016.1218252, Chinese Education \& Society, 49(3), 139151 (2016)

Zhimin, G., C. Zhongpeng y T. Jin, Empirical Study of the Role of Government Support and Success Factors in IndustryUniversity-Institute Cooperation, doi: 10.1080/10611932.2016.1218249, Chinese Education \& Society, 49 (3), 166-181 (2016) 
